\title{
On The Spot: An Android Application for Personal Safety, Location Based Alarms and Location Based Reminders
}

\author{
Nishi Tiku \\ HOD, MCA \\ VESIT, Chembur \\ Mumbai, India
}

\author{
Neha Menon \\ Department of MCA \\ VESIT, Chembur \\ Mumbai, India
}

\author{
Dhanashri Phatak \\ Department of MCA \\ VESIT, Chembur \\ Mumbai, India
}

\author{
Venkatesh \\ Vaasudhevan \\ Department of MCA \\ VESIT, Chembur \\ Mumbai, India
}

\begin{abstract}
The advancement in technology has brought with it a wide range of resources one can use to make life easier. One such resource is the Global Positioning System (GPS). Knowing the location can come in handy while providing a lot of services. The main problem while travelling is missing one's destination because of falling asleep or not knowing when to get down. The recent onslaught of violence against women and children on the road is a cause for great concern. With the increasing amount of issues, one has to face day to day, normal things like buying groceries or medicines take a backseat. Though there are applications that use location for providing services, these are still few. Smart phones are a constant in the life of most of the masses. It is easy to obtain one's location and use it to keep one safe or to provide some utility. On The Spot (OTS) is an application that does exactly the thing. It has three modules that will make the user's life more efficient and smooth. The first module, 'Locale Alarms', will allow the user to set alarms on the basis of location instead of a set time. On the other hand, 'Safety Sure', the second module is designed specifically keeping in mind the adage 'Better Safe than Sorry'. It will give periodic updates of the user's location to their loved ones without having to press an SOS button. The final module viz. 'Locale Prompts', is a 'to do' list that will allow the users to add their task and a location associated with the task; on reaching that location the user will be alerted or reminded about their task.
\end{abstract}

\section{General Terms}

Android, PhoneGap, GPS, Safety, Alarms, Reminders, Location, Travelling, Alerts

\section{Keywords}

OTS, Photo Matrix, Safety Sure, Locale Alarms, Locale Prompts

\section{INTRODUCTION}

The location is one of the most underused of the resources that are available. It has a lot of unexplored potentials. 'On The Spot' taps in on some of these areas of application. The main aim behind this research is to try and make commuting efficient and safer for people all the while using factors and tools like location and GPS, which are easily available. There has been a lot of cases wherein commuters miss stops or their destinations because of a wide array of reasons; being unaware of surroundings due to crowds or sleep and travelling for the first time are some of them. This causes a lot of hassle such as wastage of time and money while trying to get back to the original destination. Time based alarms rarely help with the aforementioned problems while travelling. The time needed to transit from a place to another is unpredictable and is affected by a lot of variables like traffic and signals. Using location for setting up alarms will give more accurate results. 'Locale Alarms' in OTS is a module which does exactly that. Another common problem that people face is important errands slipping from the minds due to the whirlwind of thoughts it holds, errands like buying groceries and/or medicines which are important but which are not considered a priority. 'Locale Prompts' is designed keeping this penchant of modern humans in mind. This module reminds the user about things they are supposed to do or buy when they reach a particular place along with a map showing them places from where they can buy the same. In the $21^{\text {st }}$ century, women are at par with men in every field. That is not to say that there are no roadblocks in their path, one of them is the increasing concern about their safety. There are many applications that are developed in the light of recent attacks on women. 'Safety Sure' module in OTS is an attempt at trying to make the roads safe for the female population. This module, on being switched on, gives periodic updates to the recipients, thus keeping them aware of the user's location and allowing them to know when they are in danger.

\subsection{Relative Work}

A through study of various systems, their literature and further analysis of their functionalities were conducted with respect to motives of OTS: safety, proximity awareness and live location alerts. Following are few systems which were studied upon:

1. 'Google Now': It is an android application that offers an integrated alarm for saved locations like home and work. The alarm knows where and approximately when you should exit and it will alert you before you get there. The reason it doesn't support OTS's motive is that the destination locations are limited: home and work. Moreover, the user cannot customize certain parameters like the distance and time metrics at which the user would like to get alerted. [1]

2. 'Watch Over Me': It is an android application that offers personal safety at a very close level to the user. It takes various inputs from the user like the event or activity which the user is performing, the estimated time that the performed activity by the user will get finished. If the user does not end his/her activity at the specified time, then the application would send emergency alerts to the designated contacts. Again, this functionality of the app doesn't support one of OTS's motives "precaution is better than cure". Such alerts would only inform the 
recipients after the event has ended, but not before the event takes place. To add upon, it also lacks customization of alerts based on distance intervals to be set by the user. [2]

3. 'Google Keep': It is an android application that offers to create location-based reminders, with a list of items. User can add reminders to notes, list items, voice recordings, and images. Other than location-based reminders, the app also provides time-specific alerts. [3]

The above mentioned existing systems are not very customizable. They are also not very flexible to the users' specific requirements. It is also learned that the user interfaces of several such apps are too cluttered, not simple to navigate and also quite difficult for naïve users to grasp the functionality of the application. The research on applications like Cheeka[4] and Abhaya[5] has led to the inference that the safety applications available till date rely on the occurrence of an undesirable event. The above survey concluded that there is no mobile application as of the date that has user-end customizations, unconditional safety measures, proximity awareness and live location alerts together in a single application which makes the proposal 'On The Spot' unique on its own.

\section{PROPOSED SYSTEM}

\section{OTS is to contain mainly three modules:}

1. Location Based Alarms: This feature allows the user to set alarms on the basis of locations. The user might know the average time to reach a place, but it is not highly accurate; the irregular traffic in case of roadways and the wait for signals in case of trains may cause the estimated time required to reach a location and the actual time to vary drastically. But if the alarms are set based on locations instead of time, it will blare exactly when at the position set, independent of aforementioned traffic or signals. It is not saying that time is not considered for the alarms. The user has the privilege of selecting a time such that the alarm will blare with that minutes to spare from the destination.

2. Location Based Reminders: Location based reminders notifies the user about establishments nearby that might help them to fulfill some of their duties from the 'to do' list. For example: If the 'to do' list contains buy medicines, the app will notify the user when there is a medical store nearby i.e. it acts like the user's portable personal assistant.

3. Safety Sure: Nowadays one cannot be too safe and safety comes in numbers. OTS will provide a safety feature that will allow the user to select recipients who will receive Short Message Service (SMS) notifications at regular intervals intimating the location of the said user. This will be very utile for users, mostly senior citizens, who have no knowledge about a place and are traversing it alone or for women, to keep their loved ones updated on their location. It also allows to know the user's exact location using the Google Maps link sent via SMS.

\subsection{Key Features}

1. The registration process involves 'Photo Matrix' and 'One Time Password' verification. The Photo Matrix is then used for authentication purposes whenever the settings are changed and also while 'Safety Sure' module is used so that unintended receivers won't get location updates.
2. In the 'Safety Sure' module, it is not a compulsion that the receiver should have internet access as the SMS would contain not only the Google Maps URL but also the actual location. This location is generated using Reverse Geocoding. [6]

3. The 'Locale Alarms' module allows users to set checkpoints at various intervals, facilitating awareness about their current position.

4. 'Locale Prompts' reminds the end user of the chores to be done as well as provides them information about all the places around them, where this job can be done along with the approximate time required to reach each option.

5. The location of the user is not stored in the server. It is stored locally on the user's device using localForage which provides persistent storage across devices running on different hardware configurations and operating systems. [7]

6. For 'Locale Alarms' and 'Safety Sure', the user has the added advantage of choosing 'when' or 'where' they want the alarms to ring or want the recipient to be notified. This can be done by the 'Distance and/or Time Interval' in case of 'Locale Alarms' and 'Distance and/or Time Alert' for 'Safety Sure'.

7. The Google Maps Distance Matrix API is used to provide the approximated distance and travelling time in the 'Locale Alarms' and 'Locale Prompts' module.

\section{WORKFLOW AND RESULTS}

\subsection{Workflow of Proposed System}

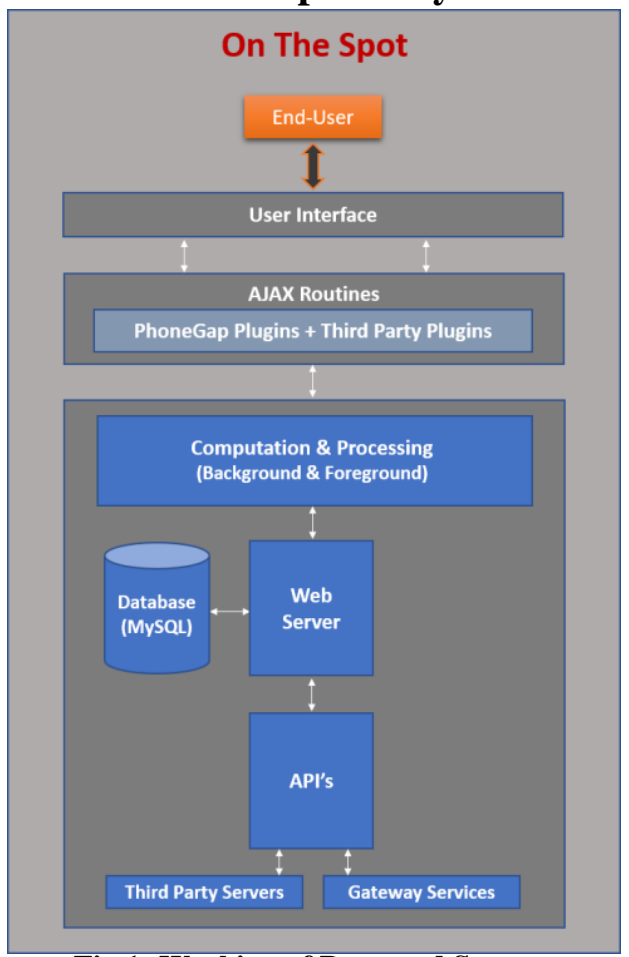

Fig 1: Working of Proposed System

The evaluation of the whole project is done module wise. There are four major modules:

Personal Details: The user has to first register for the application. An OTP is sent to the user's device for the Verification of the mobile number; on entering the correct OTP, the user is asked to login. In case of first time login, the 
user has to enter his/her date of birth, which will be used to generate demographic reports. A second layer of security is provided using Photo Matrix, whereby the user has to select two out of the set of images displayed on the screen. These values of the images are then stored to be used on a later date when the user wants to make changes to the profile information or while adding recipients in the Safety Sure module. After the Photo Matrix, the user is taken to the dashboard which has the summary of the alarms, prompts and safety sure instances currently in use.

Locale Alarms: For adding an alarm, the user has to select the destination, checkpoints if any, and the distance and/or time alert. The alarm will blare when the user reaches any one of the checkpoints and when the user is at a distance whose difference from the destination is same as the interval set. The time interval set will result in the ringing of the alarm approximately that units of time before the destination is reached. There is a limit to the number of checkpoints that can be added.

Locale Prompts: In this module, the user is supposed to populate a list of items or 'to-do' tasks and can also categorize this list according to items or tasks. The user can also set a time period after which OTS will start its ingenious process. This process starts with searching the proximity of the device with the categories mentioned by the user and locates establishments nearby that caters to these items or tasks. The user is shown alternatives that are viable out of which one can be selected on the basis of the distance and time needed to get there. It also prompts the user how to get to these establishments.

Safety Sure: To add a Safety Sure alarm, the user must fill in the time interval and/or distance interval value. Distance interval is a value in meters. When the distance travelled is equal to the value of the distance interval, an alert will be sent to the recipients. Similarly, a time interval is a value in minutes. When the time elapsed is equal to the time interval, the recipient gets an alert. The recipients' name and contact number should also be added. The alarm can be given a name based on the type of recipients. A notification is sent via SMS every time the distance interval and / or the time interval is realized. This SMS contains the actual location which is generated using Reverse Geocoding, sender's name and mobile number, the time elapsed, the distance covered and a URL which links to Google Maps.

All of the above alarms can be enabled or disabled using the toggle button.

\subsection{Result Analysis}

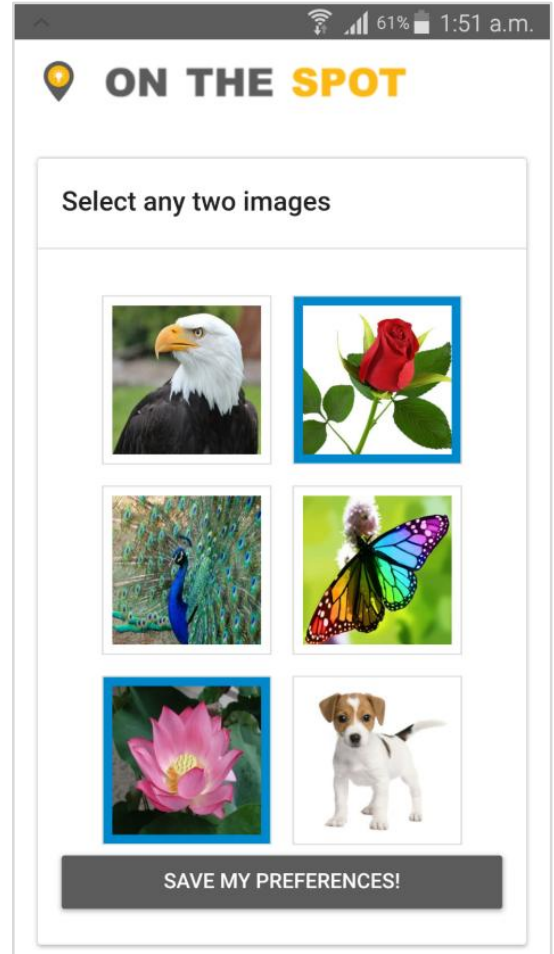

Fig 2: Photo Matrix - The user has to select two images out of the given six images for registering Photo Matrix preferences

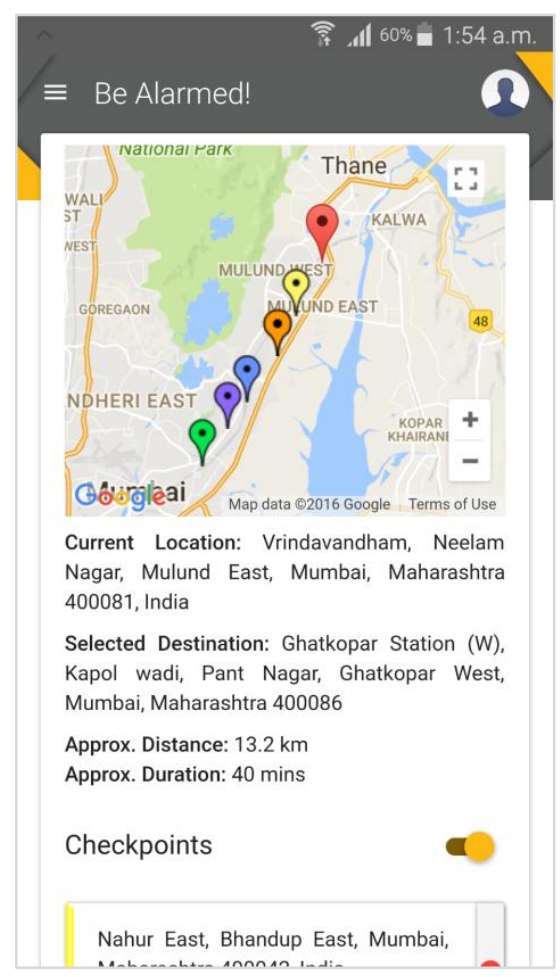

Fig 3: Locale Alarms - Checkpoints keeps the user alerted by knowing surrounding proximities 


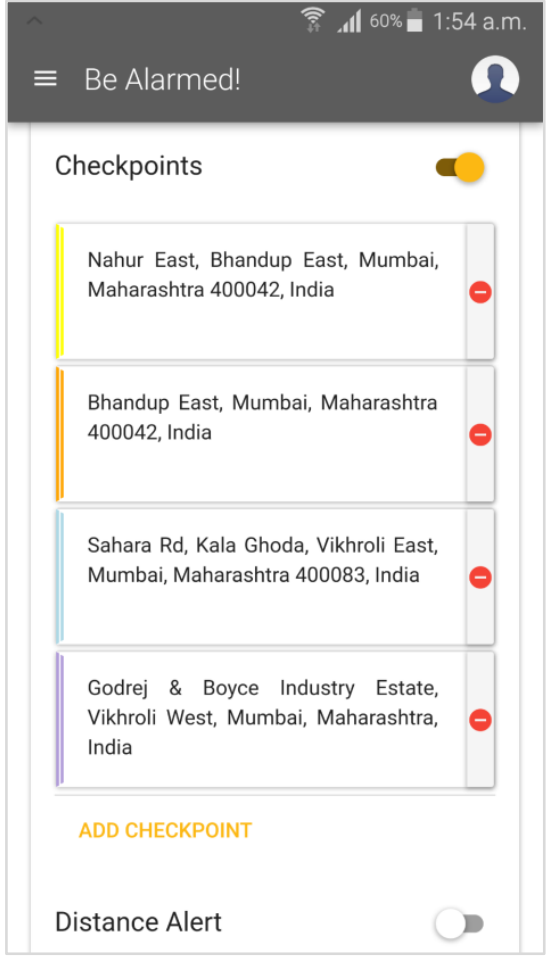

Fig 4: Locale Alarms - User can add up to a maximum of four checkpoints by repositioning the markers and can be identified by distinctive colors

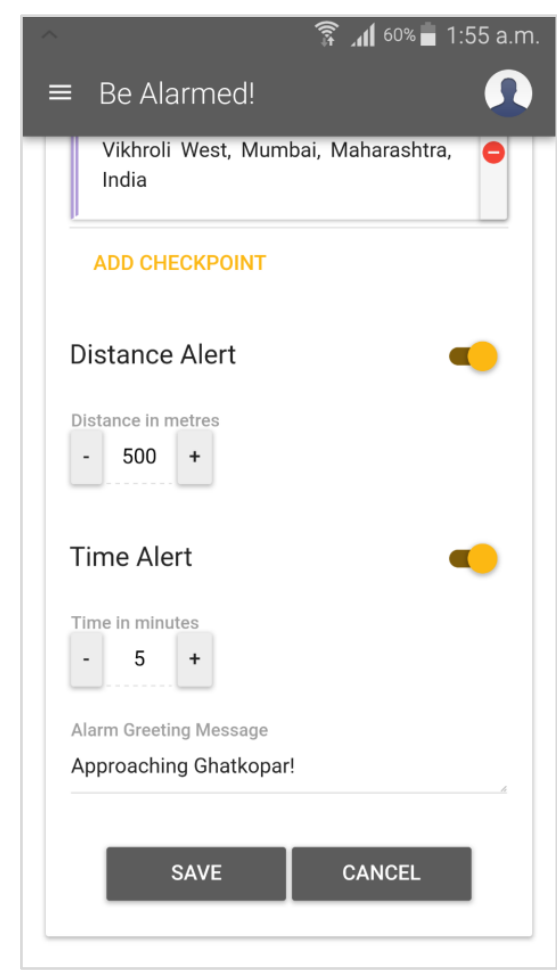

Fig 5: Distance Alerts \& Time Alerts can be set by the user along with a greeting message

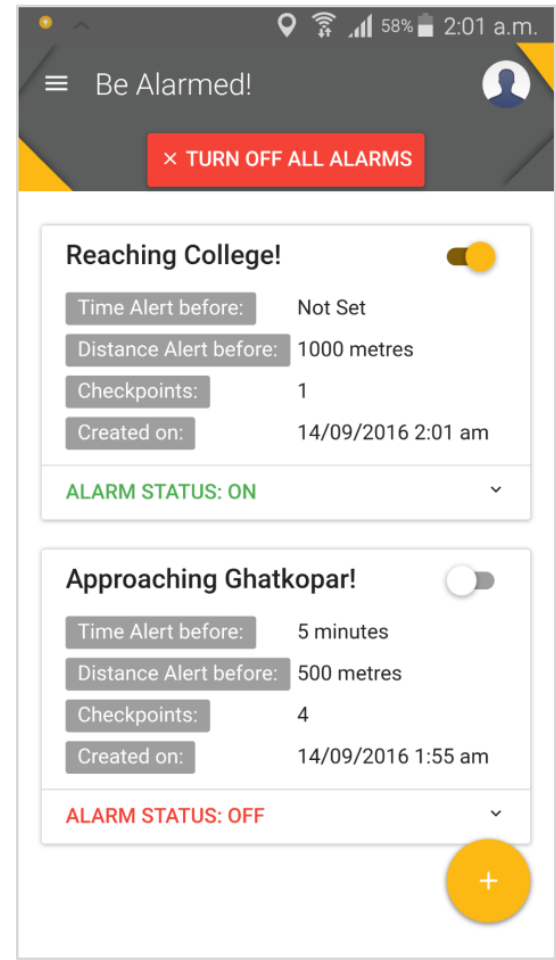

Fig 6: Locale Alarms Dashboard shows all alarms created by the user

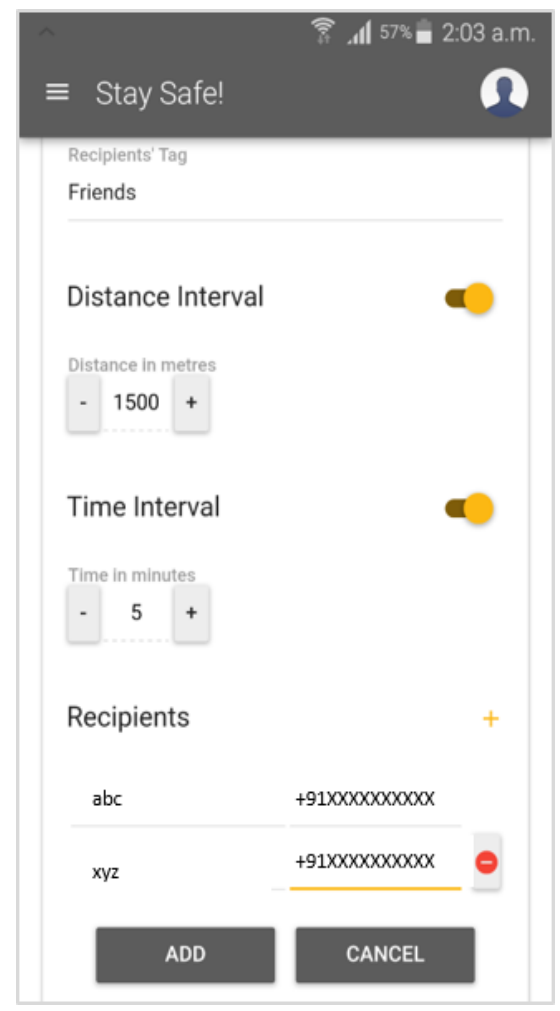

Fig 7: Safety Sure - User can add/delete recipients who will receive location updates 


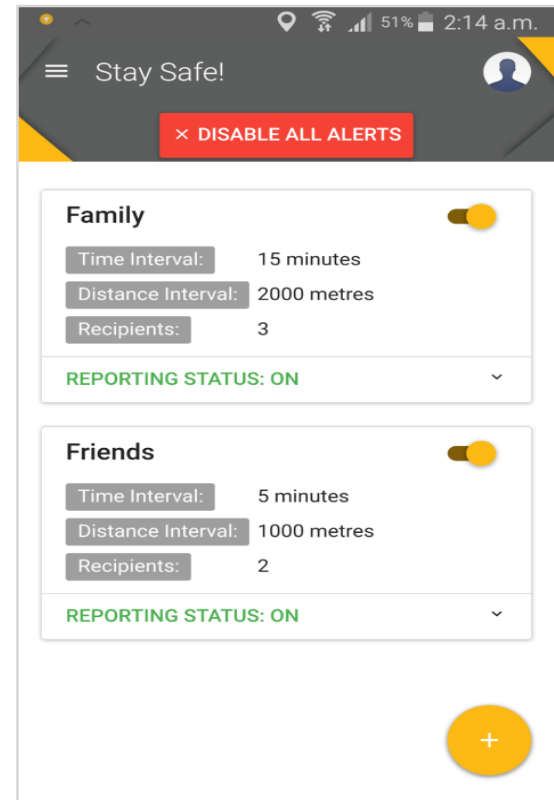

Fig 8: Safety Sure Dashboard shows all alarms created by the user

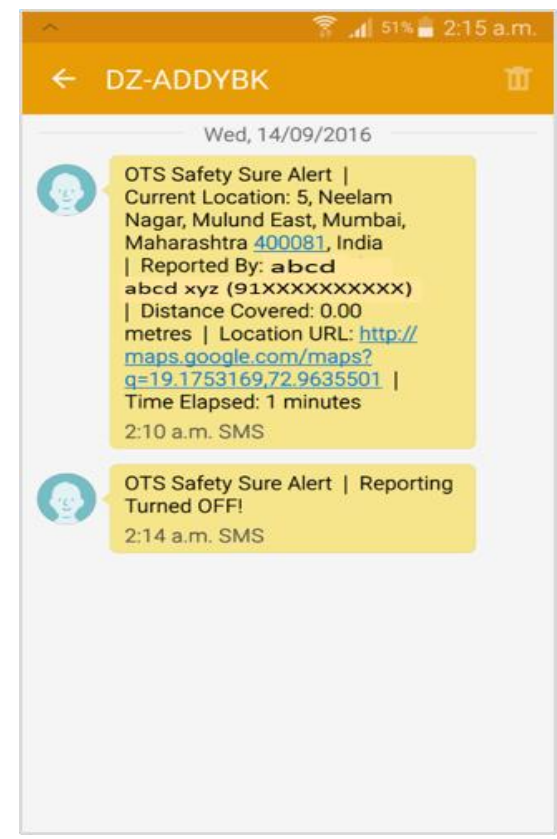

Fig 9: SMS Alert sent to recipients according to distance/time intervals provided by the user. Turn OFF SMS alerts also sent to recipients when the user turns off the alert

\section{UNIQUENESS}

While other applications provide a single functionality, OTS has combined functionality of three applications, thus reducing the number of installations. Also, the application can be used by laymen who do not have any technical knowledge. The messages sent to the recipients in Safety Sure have the actual location. Security issues too have been looked at in this application. Multiple alerts can be created in Safety Sure, wherein the recipients can vary for e.g. friends, family, acquaintances etc. can have their own alerts.

\section{CONCLUSION}

On The Spot have the cumulative features of multiple applications in one. OTS was an effort to help women feel safer as well as to make life more convenient and easy for the users. It is not to say that the project has reached its full potential. There is scope for the project to be improved tremendously. Maintenance of OTS is not a daunting task in itself. The app is quite straightforward and should be easy to maintain and keep tabs on. The cost of a system is dependent on various factors like complexity, scale of the project, UI design, device type etc.

\subsection{Future Scope}

OTS, being one of its kind applications, has a lot of scope for improvement. For one, in Safety Sure, adding recipients can be made easier by syncing it with the contacts from the phone. Again, in Safety Sure, when the battery status is beyond critical, the intended recipients can be notified of the same. The checkpoint limits can be increased for 'Locale Alarms'. Live updates of movements can be viewed on the maps. When the checkpoint is reached, the user can be shown the address of the checkpoint, the number of checkpoints left and the distance from that particular checkpoint to the destination. The battery usage of the app can be reduced. This app can be made cross platform compatible such that it works with operating systems like iOS, Windows etc. The whole project can be implemented using Internet of Things (IoT) in which a device can be developed which caters specifically to the app. This will ensure that the phone and the device will work independently and thus the usage of one won't affect the other Example: In case of battery usage.

\section{ACKNOWLEDGEMENTS}

We are grateful to all the faculty members of the Master of Computer Applications Department of V.E.S. Institute of Technology (VESIT), Mumbai for their continuous support and valuable suggestions.

We express our thanks to all our colleagues who constantly supported us without any hesitation and encouraged us during the course of our project work.

Lastly and most importantly, we are grateful to our parents for their unconditional love, constant encouragement and reassurance which gave us the strength to complete this project.

\section{REFERENCES}

[1] 'Google Now' https://play.google.com/store/apps/details?id=com.googl e.android.launcher\&hl=en

[2] 'Watch Over Me': https://play.google.com/store/apps/details?id=com. watch overme\&hl=en

[3] 'Google Keep' https://play.google.com/store/apps/details?id=com.googl e.android.keep\&hl=en

[4] Ananda Kanagaraj S, Arjun G, Shahina A. 2013. Cheeka: A mobile application for personal safety.

[5] Ravi Sekhar Yarrabothu, Bramarambika Thota. 2015. ABHAYA: AN ANDROID APP FOR THE SAFETY OF WOMEN.

[6] Reverse https://en.wikipedia.org/wiki/Reverse_geocoding

[7] 'Mozilla https://mozilla.github.io/localForage
Geocoding:

localForage': 